\title{
Parietal association deficits in patients harboring parietal lobe gliomas: a prospective study
}

\author{
Evangelia Liouta, MSc, PhD,1,2 George Stranjalis, MD,,2 Aristotelis V. Kalyvas, MD,,1,2 \\ Christos Koutsarnakis, MD, ${ }^{1,2}$ Stavroula Pantinaki, MSc, ${ }^{2}$ Faidon Liakos, MD, ${ }^{1,2}$ \\ Spyros Komaitis, MD, ${ }^{1,2}$ and Lampis C. Stavrinou, MD, PhD²
}

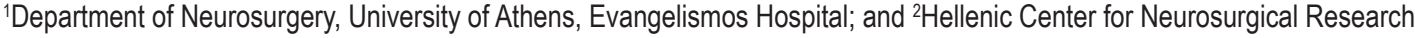
"Prof. Petros Kokkalis," Athens, Greece
\end{abstract}

OBJECTIVE Although the parietal lobe is a common site for glioma formation, current literature is scarce, consists of retrospective studies, and lacks consistency with regard to the incidence, nature, and severity of parietal association deficits (PADs). The aim of this study was to assess the characteristics and incidence of PADs in patients suffering from parietal lobe gliomas through a prospective study and a battery of comprehensive neuropsychological tests.

METHODS Between 2012 and 2016 the authors recruited 38 patients with glioma confined in the parietal lobe. Patients were examined for primary and secondary association deficits with a dedicated battery of neuropsychological tests. The PADs were grouped into 5 categories: visuospatial attention, gnosis, praxis, upper-limb coordination, and language. For descriptive analysis tumors were divided into high- and low-grade gliomas and also according to patient age and tumor size.

RESULTS Parietal association deficits were elicited in $80 \%$ of patients, thus being more common than primary deficits (50\%). Apraxia was the most common PAD (47.4\%), followed by anomic aphasia and subcomponents of Gerstmann's syndrome (34.2\% each). Other deficits such as hemineglect, stereoagnosia, extinction, and visuomotor ataxia were also detected, albeit at lower rates. There was a statistically nonsignificant difference between PADs and sex $(72.2 \%$ males, $85 \%$ females) and age $(77.8 \%$ at $\leq 60$ years, $80 \%$ at age $>60$ years), but a statistically significant difference between the $>4 \mathrm{~cm}$ and the $\leq 4 \mathrm{~cm}$ diameter group ( $p=0.02,94.7 \%$ vs $63.2 \%$, respectively). There was a tendency $(p=0.094)$ for low-grade gliomas to present with fewer PADs (50\%) than high-grade gliomas (85.7\%). Tumor laterality showed a strong correlation with hemineglect ( $p=0.004$, predilection for right hemisphere), anomia $(p=0.001)$, and Gerstmann's symptoms ( $p=0.01$ ); the last 2 deficits showed a left (dominant) hemispheric preponderance.

CONCLUSIONS This is the first study to prospectively evaluate the incidence and nature of PADs in patients with parietal gliomas. It could be that the current literature may have underestimated the true incidence of deficits. Dedicated neuropsychological examination detects a high frequency of PADs, the most common being apraxia, followed by anomia and subcomponents of Gerstmann's syndrome. Nevertheless, a direct correlation between the clinical deficit and its anatomical substrate is only possible to a limited extent, highlighting the need for intraoperative cortical and subcortical functional mapping.

https://thejns.org/doi/abs/10.3171/2017.12.JNS171799

KEYWORDS parietal lobe; gliomas; deficits; neuropsychology; neurooncology; oncology

$\mathrm{T}$ HE goal of modern neurooncological surgery is to achieve maximal tumor resection while preserving neurological function and, therefore, optimal perioperative management is of paramount importance. In this context, documentation of neuropsychological deficits through a comprehensive clinical examination in patients harboring gliomas is now considered an independent prog- nostic factor both in the extent of survival and in quality of life after tumor resection. ${ }^{16,17,20}$ In keeping with this, the current literature has focused on higher-order deficits during the preoperative neuropsychological assessment in patients with glioma, in relation to the location and extent of the lesion involved.

Although there is extensive literature on neurological

ABBREVIATIONS DTI = diffusion tensor imaging; $H G G=$ high-grade glioma; $L G G=$ low-grade glioma; $P A D=$ parietal association deficit; SLF = superior longitudinal fasciculus.

SUBMITTED July 24, 2017. ACCEPTED December 11, 2017.

INCLUDE WHEN CITING Published online May 4, 2018; DOI: 10.3171/2017.12.JNS171799. 
deficits caused by lesions in the parietal lobe, the amount of sound data with regard to parietal lobe gliomas and their relevant higher-order deficits encountered in the preoperative neurological examination through a battery of dedicated neuropsychological tests is scarce. Although the parietal lobe is a common site for glioma formation and serves as a strategic converging cerebral area for integration of multimodality information (sensory, acoustic and/or linguistic, and visual stimuli), current literature, consisting of limited retrospective studies, lacks consistency with regard to the incidence, nature, and severity of neuropsychological parietal association deficits (PADs) in patients harboring parietal gliomas. Thus, some authors focus on PADs, i.e., Gerstmann's syndrome and sensory association disturbances, reporting that patients did not suffer from aphasia, while others support the claim that language deficits are the most common association symptom, whereas Gerstmann's syndrome is rare. ${ }^{23,24}$

Hence, our objective was to shed light on this issue by thoroughly assessing the unique characteristics of parietal lobe higher-order deficits in patients suffering from parietal lobe gliomas through a prospectively organized study and a comprehensive neuropsychological examination. To our knowledge this is the first prospective study that has focused on this specific neuropsychological topic.

\section{Methods}

The study was conducted according to the guidelines of the Helsinki Declaration, and permission was granted by the local ethics committee. Informed consent was obtained from all patients or their legal guardians prior to their inclusion in the study. Inclusion criteria were 1) patients with newly diagnosed parietal lobe glioma, and 2) good knowledge of the Greek language, so that the neuropsychological assessment would be valid. Exclusion criteria were 1) previous treatment, 2) history of intracranial mass lesion, 3) history of traumatic brain injury, and 4) history of other CNS diseases (e.g., stroke).

Thirty-eight consecutive patients harboring a primary confined parietal lobe glioma for which resection was planned were prospectively recruited over a 4-year period (2012-2016) from a single institution (Department of Neurosurgery, "Evangelismos" Hospital, Athens, Greece). Tumor location was verified using standard multiplanar MR images. The classic anatomical landmarks-namely the central sulcus anteriorly, the parieto-occipital fissure posteriorly, and the posterior part of lateral sulcus inferolaterally, were identified as the boundaries of the parietal lobe. The tumor had to be located within those confines. All patients were examined for parietal lobe secondary association deficits during preoperative neuropsychological assessment by the first author. Primary sensorimotor (hypo- or hyperesthesia and/or paresis) and visual deficits (hemianopia) were examined by 2 senior neurosurgical residents. Clinical examination was conducted approximately 7 days prior to resection.

Secondary association deficits were grouped into 5 categories: 1) visuospatial attention, 2) gnosis, 3) praxis, 4) upper-limb coordination, and 5) language. Deficits in visuospatial attention included visual contralateral hemine- glect, double visual extinction, and double tactile extinction. Visual hemineglect was assessed through the line bisection task and Balloons cancellation test for visual inattention..$^{10,25}$ Visual and tactile extinction was evaluated by applying unilateral and bilateral simultaneous sensory stimulation (visual and tactile, respectively) in a randomly alternative order for 3 consecutive trials. Deficits in gnosis included stereoagnosia, somatoagnosia, and finger agnosia (a manifestation of Gerstmann's syndrome). Stereoagnosia was investigated by asking blindfolded patients to recognize objects stereotactically, e.g., coin, key, and pen, whereas somatoagnosia and finger agnosia were investigated by asking patients to name or point to specific parts of their body and to name their own or the examiners' finger, respectively. Deficits in praxis included constructional apraxia, ideomotor apraxia, and ideational apraxia. With regard to the first type we asked patients to draw a clock, whereas for the 2 latter ones we used the apraxia test developed by Zwinkels et al. ${ }^{32}$ This specific test consists of 2 subtests: demonstration of object use, which evaluates ideomotor apraxia; and imitation of gestures, which assesses ideational apraxia. For ideomotor apraxia 9 tasks were applied: 3 verbal commands with no objects presented (e.g., "Can you show me how you would use a key?"); 3 verbal and visual commands with the objects placed in front of the patients but without their being allowed to touch them (e.g." "Can you show me how you would use the spoon? You cannot touch it."); and 3 tasks in which the patient was asked to use an object (e.g., a screwdriver) without naming it. With regard to ideational apraxia, 6 gestures (e.g., saluting, making a fist) were demonstrated as mirror images and the patient was invited to imitate the examiner. Coordination deficit was defined as visuomotor ataxia of the upper limb and assessed by the finger-nose-tapping task. Language deficits included nominal aphasia (anomia), alexia or dyslexia, and agraphia or dysgraphia. Nominal aphasia was assessed by the Boston Naming Test, alexia by asking patients to read aloud a text, and agraphia by asking them to write a sentence. ${ }^{15}$ Finally, acalculia and left-right confusion, as parts of Gerstmann's syndrome, were also prospectively examined.

Patients with motor weakness or sensory loss were not assessed for apraxia and tactile extinction and/or stereoagnosia, respectively, because to reliably diagnose a deficit as a secondary association, the relevant primary function needs to be intact.

For descriptive analysis, tumors were divided into 1) high-grade gliomas (HGGs), which included glioblastoma, anaplastic astrocytoma, anaplastic oligodendroglioma, and anaplastic mixed glioma; and 2) low-grade gliomas (LGGs), including WHO grade II astrocytoma, oligoastrocytoma, and oligodendroglioma.

\section{Statistical Analysis}

Data were analyzed using the SPSS software package (IBM SPSS Statistics for Windows, version 21; IBM Corp.). Statistical analysis showed normal distribution of data. Fisher exact tests were performed to examine the relationship between demographic data, namely age (2 groups: 29-60 years, and 61-79 years) and sex, tumor diameter ( $>4 \mathrm{~cm}$ and $\leq 4 \mathrm{~cm}$ ), laterality, grade (LGG and HGG), and PADs. 


\section{Results}

Demographic and clinical data at time of presentation are listed in Table 1, as are tumor basic characteristics. Sex was equally distributed across the sample, with patients having a fair level of education and being right-handed according to the Edinburgh Handedness Inventory, except for 1 patient who was left-handed. ${ }^{21}$ The most commonly presenting symptoms were seizures (34\%), followed by cognitive changes (i.e., memory, concentration disturbance ([26.3\%]), and muscle weakness (21\%). Gliomas were almost equally distributed between the 2 hemispheres, with the majority of them being HGGs histopathologically (84.2\% HGG vs $15.8 \%$ LGG).

\section{Incidence of Deficits}

Approximately $50 \%$ of patients presented with primary deficits such as contralateral weakness, hypesthesia, and visual field deficits. Secondary parietal deficits were elicited in $80 \%$ of patients, thus proving to be more common than primary ones. More specifically and in terms of frequency, apraxia was the most common PAD - seen in $47.4 \%$ of patients. Language deficits and subcomponents of Gerstmann's syndrome were rated in second place with

TABLE 1. Characteristics of 38 patients with parietal glioma at presentation

\begin{tabular}{|c|c|}
\hline Characteristic & No. of Patients (\%) \\
\hline \multicolumn{2}{|l|}{ Patient } \\
\hline \multicolumn{2}{|l|}{ Sex } \\
\hline Male & $18(47.4)$ \\
\hline Female & $20(52.6)$ \\
\hline \multicolumn{2}{|l|}{ Handedness } \\
\hline Rt & $37(97.4)$ \\
\hline $\mathrm{Lt}$ & $1(2.6)$ \\
\hline \multicolumn{2}{|l|}{ Age in yrs } \\
\hline Mean \pm SD & $59.7 \pm 13.2$ \\
\hline Range & $29-79$ \\
\hline \multicolumn{2}{|l|}{ Education in yrs } \\
\hline Mean \pm SD & $10.8 \pm 3.8$ \\
\hline Range & $4-16$ \\
\hline \multicolumn{2}{|l|}{ Symptom at presentation } \\
\hline Seizure & $13(34.2)$ \\
\hline Headache & $6(15.8)$ \\
\hline Weakness & $8(21.1)$ \\
\hline Mental alteration & $10(26.3)$ \\
\hline No symptom & $1(2.6)$ \\
\hline \multicolumn{2}{|l|}{ Tumor } \\
\hline \multicolumn{2}{|l|}{ Histology } \\
\hline LGG & $6(15.8)$ \\
\hline HGG & $32(84.2)$ \\
\hline \multicolumn{2}{|l|}{ Laterality } \\
\hline $\mathrm{Lt}$ & $21(55.3)$ \\
\hline Rt & $17(44.7)$ \\
\hline Mean diameter, range in $\mathrm{cm}$ & $4.7,2.3-7$ \\
\hline
\end{tabular}

an almost equal distribution of $34.2 \%$, and were followed (in order) by visuospatial contralesional hemineglect, stereoagnosia, double visual and tactile extinction, somatoagnosia, and visuomotor ataxia. Table 2 shows the frequency of parietal deficits in our sample.

\section{Demographics and PADs}

Results indicate a nonsignificant difference $(\mathrm{p}=0.28)$ in the presence of PADs between males $(13 / 18,72.2 \%)$ and females $(17 / 20,85 \%)$. Regarding age, results also showed no statistically significant difference $(\mathrm{p}=0.58)$ between younger age (29-60 years old, prevalence $77.8 \%$ ) and older age (61-79 years old, prevalence $80 \%$ ) groups. When the effect of demographics on each deficit was analyzed separately, age was shown to have an effect $(\mathrm{p}=0.031)$ on ideomotor apraxia, with the older age group being affected more frequently ( 7 of 20 patients, 35\%) when compared with the younger age group $(1 / 18,5.6 \%)$. There was no statistical significance between demographic variables and other deficits.

\section{Tumor Characteristics and PADs}

In relation to tumor size, results indicated a statistically significant difference $(\mathrm{p}=0.02)$ between the $>4 \mathrm{~cm}$ diameter group (prevalence 94.7\%, 18/19 patients) and the $\leq 4$ $\mathrm{cm}$ diameter group $(63.2 \%, 12 / 19)$ concerning PADs. With regard to tumor histology, although results did not reach

TABLE 2. Frequency of primary and nonprimary (secondary or association) deficits

\begin{tabular}{lc}
\hline \multicolumn{1}{c}{ Deficits } & Prevalence of Deficit (\%) \\
\hline Primary deficits & $6(15.8)$ \\
\hline Visual field loss & $12(31.6)$ \\
\hline Weakness-hypesthesia & \\
\hline Nonprimary deficits & $8(21.1)$ \\
\hline Absent & $30(78.9)$ \\
\hline Present & $5(13.2)$ \\
\hline Visual extinction & $5(13.2)$ \\
\hline Tactile extinction & $6(15.8)$ \\
\hline Hemineglect & $3(7.9)$ \\
\hline Somatoagnosia & $6(15.8)$ \\
\hline Stereoagnosia & $13(34.2)$ \\
\hline Gerstmann's (total) & $6(15.8)$ \\
\hline Gerstmann's syndrome (complete) & \\
\hline Gerstmann's partial components & $1(2.6)$ \\
\hline Finger agnosia & $3(7.9)$ \\
\hline Acalculia & $3(7.9)$ \\
\hline Agraphia & $18(47.4)$ \\
\hline Apraxia (total) & $5(13.2)$ \\
\hline Ideational & $8(21.1)$ \\
\hline Ideomotor & $5(13.2)$ \\
\hline Constructional & $13(34.2)$ \\
\hline Anomia & $2(5.3)$ \\
\hline Ataxia &
\end{tabular}


statistical significance $(p=0.094)$, there was a tendency for the LGG group to present with fewer PADs (3/6 patients, 50\%) when compared with the HGG group (27/32, 84.4\%). However, the small size of the LGG group does not allow for a definite conclusion to be drawn. Finally, with respect to tumor laterality, results indicated a nonsignificant difference $(p=0.23)$ in the overall presence of PADs between patients with a left hemispheric $(18 / 21,85.7 \%)$ and a right hemispheric (12/17, 70.6\%) glioma. When the effect of tumor characteristics on each deficit was analyzed, tumor laterality had a statistically significant correlation with hemineglect $(\mathrm{p}=0.004)$, which presented exclusively in patients with a right parietal lesion $(35.3 \%, 6 / 17)$. Additionally, results disclosed a statistically significant difference in anomia $(\mathrm{p}=0.001)$ between left $(12 / 21,57.1 \%)$ and right $(1 / 17,5.9 \%)$ parietal lobe pathologies. Finally, tumor laterality had a statistically significant difference $(p=0.01)$ in Gerstmann's syndrome (9/21 [42.9\%] for left vs 1/17 [5.9\%] for right parietal lesions). Of note is the fact that the patient with the right hemispheric lesion who presented with anomia and Gerstmann's syndrome was the same individual, and the tumor-carrying hemisphere was the dominant one. Tumor histology and tumor size did not have a statistically significant effect on individual PADs.

\section{Discussion}

In the field of neurosurgical oncology, focused neuropsychological assessment of higher cognitive functions and their putative deficiency due to glial tumors has become increasingly prominent in the preoperative planning and postoperative management of neurooncology patients. Toward this direction, most of the published data have mainly focused on frontal and temporal lobe higher-order functions (e.g., executive functions, language, speech, limbic functions, and so on), while the parietal lobe has been so far relatively underrated. Apart from a few case series, current neurosurgical literature consists of 2 retrospective studies that provide conflicting evidence with regard to the nature and incidence of glioma-associated secondary parietal lobe deficits. ${ }^{23,24}$ To our knowledge, this is the first prospectively organized study aiming to evaluate and report the incidence of nonprimary deficits through a tailored battery of neuropsychological tests in patients harboring parietal lobe gliomas.

Interestingly enough, the majority of patients enrolled (approximately $80 \%$ ) presented with various PADs when examined systematically. This is consistent with previous research, highlighting the fact that neuropsychological assessment yields findings of higher-function deficits in the majority of glioma patients, even when the results of the standard neurological examination are negative, a fact particularly prominent in patients presenting with LGGs.? During our study the most common deficit encountered was that of apraxia, followed by anomic aphasia and a variety of Gerstmann's syndrome clinical manifestations.

\section{Apraxia and Parietal Lobe}

Apraxia is defined as the inability to perform certain subjectively purposeful movements or movement complexes in the absence of "elemental" motor deficits or gen- eral cognitive impairment. ${ }^{11}$ Apraxic errors can involve imitating gestures (ideational type of apraxia), performing meaningful gestures and using tools or objects (ideomotor type of apraxia), and reproducing patterns or joining component parts into a whole that can be assessed by asking patients to draw or build 3D objects (constructional apraxia). The anatomical substrate of apraxia remains as yet an equivocal issue; even though lesions located in the inferior parietal lobule and temporoparietal junction have been traditionally related to apraxic symptoms, the premotor cortex and basal ganglia have also been functionally linked to apraxia, thus adding complexity to this topic. ${ }^{29}$ Recently, an increasing amount of evidence supports the idea that "pure" parietal lobe lesions predominantly affect imitation of gestures and consequently the use of tools, whereas other types of apraxia result from more extensive cerebral damage outside the boundaries of the parietal lobe. Moreover, Buxbaum et al. have reported that patients sustaining left or dominant hemisphere damage and presenting with apraxia had a significantly higher incidence of angular gyrus disruption than nonapraxic patients. Notably, 8 of the 10 apraxic patients had difficulties imitating hand gestures, a symptom of ideational apraxia. ${ }^{5}$

With regard to the incidence of apraxia, this is the first study in the literature to report its high incidence in patients with parietal lobe gliomas. As a matter of fact, apraxia was the most common PAD encountered. In agreement with previous thought, ideomotor and ideational forms of apraxia were seen in patients with dominant parietal lesions, whereas constructional apraxia was found only in nondominant parietal lobe gliomas. When viewed as a symptom manifested mostly in clinical settings, evidence suggests that apraxia is particularly debilitating, affecting daily living,,${ }^{14}$ and is associated with dependence on caregivers for certain daily activities., ${ }^{4,5,12,28}$

\section{Anomic Aphasia and Parietal Lobe}

Anomia is defined as the inability to name visually presented objects while perception of their form and knowledge of their meaning and usage remain intact. The cerebral territory most involved in naming is the dominant inferior parietal lobule, with current literature based on intraoperative direct current stimulation confirming that angular and supramarginal areas have the highest probability of producing anomic errors when their function is temporarily disrupted. ${ }^{6,8}$ Findings from intraoperative stimulation have also shown an extreme interindividual variability regarding the localization of essential language-related areas, thus making it impossible to predict them preoperatively. ${ }^{6,19}$ The presence of anomia, however, is an indication that the arcuate fasciculus and the superior longitudinal fasciculus (SLF), i.e., the major fiber tracts involved in the dorsal stream of language processing, are affected. This fact highlights the need for subcortical mapping, especially in patients harboring LGGs, because diffusion tensor imaging (DTI) might reconstruct fibers displaced by the tumor, but may not depict fibers that are invaded but are still functional..$^{18}$ To complicate things further, the SLF, the horizontal portion of the lateral segment of the SLF (SLF III), and the arcuate fasciculus have all been shown to produce nominal aphasia when stimulated. ${ }^{6}$ 
In the neurosurgical literature, the term "aphasia" has been used broadly, and often encompasses fluency deficits, reduced information content of spoken output, repetition deficits, and nominal deficits (nominal aphasia)..$^{30}$ The preoperative identification of nominal aphasia is particularly important in glioma surgery due to the fact that, in contrast to the other speech and language deficits, which usually resolve over time, nominal aphasia seems to persist postoperatively. ${ }^{18}$ In regard to previous studies, our results provide further support to Sanai et al.'s findings, according to which anomic aphasia is a rather common deficit in patients harboring parietal gliomas. ${ }^{23} \mathrm{On}$ the other hand, our results are in disagreement with Russell et al.'s findings, according to which aphasia was not detected in their sample. ${ }^{24}$

\section{Gerstmann's Syndrome}

Gerstmann's syndrome comprises symptoms of finger agnosia, agraphia, acalculia, and right-left confusion. Ambiguity remains as to the anatomical substrate of the syndrome, but most probably it involves the left angular gyrus with its subcortical territory. ${ }^{1}$ Interestingly, a "pure" and complete form of Gerstmann's syndrome is quite rare because it usually unfolds as either an "incomplete" tetrad or is associated with other cognitive deficits, including aphasia, alexia, and some perceptual disorders. ${ }^{22}$ In a study enrolling 194 stroke patients recently published by Zukic et al., 59 had alexia, agraphia, and acalculia, or different combination of these deficits, and a complete, "pure" Gerstmann's syndrome was disclosed in only 2 patients. ${ }^{31}$ Benton has also argued that the neuropsychological deficits forming Gerstmann's syndrome did not show a stronger correlation with each other than did other combinations of deficits. ${ }^{3}$

Regarding the classic Gerstmann's syndrome, agraphia is usually the absent clinical entity: This may be due to the fact that agraphia has a more widespread anatomical variability and correspondence and has been mainly linked to the superior parietal lobule, as opposed to the angular gyrus, which seems to underpin Gerstmann's syndrome. ${ }^{1-3,13}$ Accordingly, in our study agraphia was uncommon in the so-called Gerstmann's symptoms, seen in 3 patients-and, interestingly enough, these patients belonged to the group with larger tumors $(>4 \mathrm{~cm})$. At the same time, one study with 194 patients could find only 2 patients with a "pure" Gerstmann's syndrome, because Gerstmann's symptoms were always associated with some other extraneous symptoms - the most common being aphasia, followed by apraxia. ${ }^{31}$ Therefore, in agreement with existing evidence, the 3 most common symptoms in our study were exactly those: components of Gerstmann's syndrome, apraxia, and aphasia.

With regard to previous studies, our results provide further support to Russell et al.'s findings and disagree with Sanai et al.'s study, according to which components of Gerstmann's syndrome are rarely present in patients with parietal gliomas. ${ }^{23,24}$

\section{Low-Grade Glioma Versus High-Grade Glioma}

There was a statistically nonsignificant trend for LGG to present with fewer PADs (50\%) as compared with HGG (84.4\%), although the small number of patients with LGG included in the present study does not allow for definite conclusions to be drawn. Nonetheless, several hypotheses regarding tumor histology can be made; thus, the faster growth rate of HGG may not allow for enough time for the recruitment of adjacent structures, which may compensate for deficits..$^{19}$ Moreover, the destructive growth pattern of HGG may be more deleterious to parietal lobe function than the infiltrating pattern of LGG. Such hypotheses are also relevant when considering postresection deficits; the contrast-enhancing portions of HGGs should not contain any functional tissue and are not expected to produce new deficits with their removal. On the other hand, LGGs, which may grow diffusely around areas of potentially viable tissue, could produce a higher incidence of neurological deficits postoperatively. ${ }^{26,27}$

The present study was designed prior to the new WHO classification and, therefore, tumors were categorized as LGG and HGG based primarily on their histology. The 2016 WHO classification of CNS tumors uses molecular parameters in addition to histology to define many tumor entities. Undoubtedly, more objective and precisely defined entities will allow for improved tailoring of patient therapy, prediction of prognosis, better classification for clinical trials and experimental studies, and more precise categorization for epidemiological purposes. It is unclear whether this updated taxonomy would have had an impact on our findings, given the fact that even with the older classification there were no statistically significant differences between the groups.

\section{Strengths and Limitations of Our Study}

The present study is the first prospective investigation to evaluate PADs in patients harboring parietal lobe gliomas by using a comprehensive battery of neuropsychological tests. Current relevant literature consists of only 2 retrospective studies, which report conflicting results regarding the incidence of aphasia and components of Gerstmann's syndrome. ${ }^{23,24}$ It could be that data mining of neurological examinations (with varying degrees of specificity regarding detection of symptoms) may have underestimated the true incidence of deficits. At the same time the small number of patients, some of them harboring large tumors, does not allow for a precise subgroup analysis regarding location (for example superior vs inferior parietal lobule) and histology.

Whether the detected deficits, primary or secondary, are attributable to either a cortical or a subcortical dysfunction extending beyond the parietal lobe could be not clarified in the present study. Such a differentiation would require a combination of DTI-MRI studies and intraoperative cortical and subcortical mapping during an awake craniotomy. Even with these investigations, the distinction can be challenging, especially given the fact that most tumors present with deficits caused by a combination of both kinds of dysfunction. ${ }^{9}$

\section{Conclusions}

This is the first study to prospectively evaluate the in- 
cidence and nature of PADs in patients harboring parietal gliomas. Dedicated clinical examination detects a high frequency of PADs ( $80 \%$ of patients). The most frequent deficit encountered in our patients was that of apraxia, followed by anomic aphasia and components of Gerstmann's syndrome. Insight into the preoperative deficits, especially those that may be subclinical and require a dedicated examination, helps the neurosurgeon to understand which structures are affected and-most importantly-are at additional surgical risk and may increase postoperative morbidity. Knowing that a certain function is preoperatively affected can help put imaging data (functional MRI and especially DTI) into perspective and may strengthen the indication for cortical and subcortical functional mapping.

The neuropsychological battery presented in this paper embodies a core selection of easily reproducible tests for the detection of secondary parietal deficits. Therefore, these also represent a proposal for a standardized battery of tests to examine parietal lobe function.

\section{References}

1. Ardila A: A proposed reinterpretation of Gerstmann's syndrome. Arch Clin Neuropsychol 29:828-833, 2014

2. Benton AL: Gerstmann's syndrome. Arch Neurol 49:445447, 1992

3. Benton AL: Reflections on the Gerstmann syndrome. Brain Lang 4:45-62, 1977

4. Buxbaum LJ, Haaland KY, Hallett M, Wheaton L, Heilman KM, Rodriguez A, et al: Treatment of limb apraxia: moving forward to improved action. Am J Phys Med Rehabil 87:149-161, 2008

5. Buxbaum LJ, Sirigu A, Schwartz MF, Klatzky R: Cognitive representations of hand posture in ideomotor apraxia. Neuropsychologia 41:1091-1113, 2003

6. Chang EF, Raygor KP, Berger MS: Contemporary model of language organization: an overview for neurosurgeons. J Neurosurg 122:250-261, 2015

7. Cochereau J, Herbet G, Duffau H: Patients with incidental WHO grade II glioma frequently suffer from neuropsychological disturbances. Acta Neurochir (Wien) 158:305-312, 2016

8. Duffau H: The error of Broca: from the traditional localizationist concept to a connectomal anatomy of human brain. $\mathbf{J}$ Chem Neuroanat [epub ahead of print], 2017

9. Duffau H, Capelle L, Denvil D, Sichez N, Gatignol P, Taillandier L, et al: Usefulness of intraoperative electrical subcortical mapping during surgery for low-grade gliomas located within eloquent brain regions: functional results in a consecutive series of 103 patients. J Neurosurg 98:764-778, 2003

10. Edgeworth J, Robertson I, McMillan T: The Balloons Test. Bury St Edmunds, UK: Thames Valley Test Company, 1998

11. Goldenberg G: Apraxia and the parietal lobes. Neuropsychologia 47:1449-1459, 2009

12. Hanna-Pladdy B, Heilman KM, Foundas AL: Ecological implications of ideomotor apraxia: evidence from physical activities of daily living. Neurology 60:487-490, 2003

13. Heimburger RF, Demyer W, Reitan RM: Implications of Gerstmann's syndrome. J Neurol Neurosurg Psychiatry 27:52-57, 1964

14. Jager G, Postma A: On the hemispheric specialization for categorical and coordinate spatial relations: a review of the current evidence. Neuropsychologia 41:504-515, 2003

15. Kaplan E, Goodglass H, Weintraub S: Boston Naming Test. Philadelphia: Lea \& Febiger, 1983

16. Klein M, Duffau H, De Witt Hamer PC: Cognition and resec- tive surgery for diffuse infiltrative glioma: an overview. J Neurooncol 108:309-318, 2012

17. Klein M, Postma TJ, Taphoorn MJ, Aaronson NK, Vandertop WP, Muller M, et al: The prognostic value of cognitive functioning in the survival of patients with high-grade glioma. Neurology 61:1796-1798, 2003

18. Leclercq D, Duffau H, Delmaire C, Capelle L, Gatignol P, Ducros M, et al: Comparison of diffusion tensor imaging tractography of language tracts and intraoperative subcortical stimulations. J Neurosurg 112:503-511, 2010

19. Maldonado IL, Moritz-Gasser S, de Champfleur NM, Bertram L, Moulinié G, Duffau H: Surgery for gliomas involving the left inferior parietal lobule: new insights into the functional anatomy provided by stimulation mapping in awake patients. J Neurosurg 115:770-779, 2011

20. Meyers CA, Hess KR, Yung WK, Levin VA: Cognitive function as a predictor of survival in patients with recurrent malignant glioma. J Clin Oncol 18:646-650, 2000

21. Oldfield RC: The assessment and analysis of handedness: the Edinburgh inventory. Neuropsychologia 9:97-113, 1971

22. Rusconi E, Pinel P, Dehaene S, Kleinschmidt A: The enigma of Gerstmann's syndrome revisited: a telling tale of the vicissitudes of neuropsychology. Brain 133:320-332, 2010

23. Russell SM, Elliott R, Forshaw D, Kelly PJ, Golfinos JG: Resection of parietal lobe gliomas: incidence and evolution of neurological deficits in 28 consecutive patients correlated to the location and morphological characteristics of the tumor. $\mathbf{J}$ Neurosurg 103:1010-1017, 2005

24. Sanai N, Martino J, Berger MS: Morbidity profile following aggressive resection of parietal lobe gliomas. J Neurosurg 116: $1182-1186,2012$

25. Schenkenberg T, Bradford DC, Ajax ET: Line bisection and unilateral visual neglect in patients with neurologic impairment. Neurology 30:509-517, 1980

26. Schiffbauer H, Ferrari P, Rowley HA, Berger MS, Roberts TP: Functional activity within brain tumors: a magnetic source imaging study. Neurosurgery 49:1313-1321, 2001

27. Skirboll SS, Ojemann GA, Berger MS, Lettich E, Winn HR: Functional cortex and subcortical white matter located within gliomas. Neurosurgery 38:678-685, 1996

28. Smania N, Girardi F, Domenicali C, Lora E, Aglioti S: The rehabilitation of limb apraxia: a study in left-brain-damaged patients. Arch Phys Med Rehabil 81:379-388, 2000

29. Wheaton LA, Hallett M: Ideomotor apraxia: a review. J Neurol Sci 260:1-10, 2007

30. Wilson SM, Lam D, Babiak MC, Perry DW, Shih T, Hess CP, et al: Transient aphasias after left hemisphere resective surgery. J Neurosurg 123:581-593, 2015

31. Zukic S, Mrkonjic Z, Sinanovic O, Vidovic M, Kojic B: Gerstmann's syndrome in acute stroke patients. Acta Inform Med 20:242-243, 2012

32. Zwinkels A, Geusgens C, van de Sande P, Van Heugten C: Assessment of apraxia: inter-rater reliability of a new apraxia test, association between apraxia and other cognitive deficits and prevalence of apraxia in a rehabilitation setting. Clin Rehabil 18:819-827, 2004

\section{Disclosures}

The authors report no conflict of interest concerning the materials or methods used in this study or the findings specified in this paper.

\section{Author Contributions}

Conception and design: Stavrinou, Liouta. Acquisition of data: Liouta, Kalyvas, Pantinaki. Analysis and interpretation of data: Kalyvas, Pantinaki. Drafting the article: Stavrinou, Liouta. Critically revising the article: Stavrinou, Liouta, Koutsarnakis. 
Reviewed submitted version of manuscript: Stavrinou, Liouta, Stranjalis, Koutsarnakis, Komaitis. Approved the final version of the manuscript on behalf of all authors: Stavrinou. Statistical analysis: Komaitis. Administrative/technical/material support: Stranjalis, Liakos. Study supervision: Stavrinou, Stranjalis.

\section{Correspondence}

Lampis C. Stavrinou: Hellenic Center for Neurosurgical Research "Prof. Petros Kokkalis," Athens, Greece.mplam@hotmail.com. 